\title{
Helicobacter suis induces changes in gastric inflammation and acid secretion markers in pigs of different ages
}

\author{
C. De Witte ${ }^{1 *}\left(\mathbb{D}\right.$, B. Devriendt ${ }^{2}$, B. Flahou ${ }^{1}$, I. Bosschem ${ }^{1}$, R. Ducatelle ${ }^{1}$, A. Smet $^{3 \dagger}$ and F. Haesebrouck ${ }^{1 \dagger}$
}

\begin{abstract}
Gastric mRNA expression of markers for acid secretion and inflammation and presence of gastric ulceration was studied in naturally Helicobacter suis-infected and non-infected 2-3 months old, 6-8 months old and adult pigs. In $\mathrm{H}$. suis-infected 2-3 months old pigs, IL-8 and IL-1 $\beta$ transcript levels were upregulated in the pyloric gland zone, indicating an innate immune response. A similar response was demonstrated in the fundic gland zone of adult pigs, potentially due to a shift of $H$. suis colonization from the pyloric to the fundic gland zone. A Treg response in combination with decreased expressions of IL-8, IL-17A and IFN- $\gamma$ was indicated to be present in the H. suis-infected 6-8 months old pigs, which may have contributed to persistence of $H$. suis. In H. suis-infected adult pigs, a Treg response accompanied by a Th17 response was indicated, which may have played a role in the decreased number of $H$. suis bacteria in the stomach of this age group. The decreased G-cell mass and upregulated expression of somatostatin indicated decreased acid secretion in $\mathrm{H}$. suis-infected 6-8 months old pigs. In H. suis-infected adult pigs, upregulation of most markers for gastric acid secretion and increased G-cell mass was detected. Presence of severe hyperkeratosis and erosions in the non-glandular part of the stomach were mainly seen in the H. suis-positive groups. These results show that $H$. suis infection affects the expression of markers for acid secretion and inflammation and indicate that these effects differ depending on the infection phase.
\end{abstract}

\section{Introduction}

Gastric ulceration is a common disease entity of pigs worldwide, with prevalences of up to $93 \%$ [1]. Although the disease outcome is often subclinical, animal welfare issues as well as economic losses due to decreased daily weight gain, decreased feed intake and sudden death, are of major importance [1]. The etiology seems to be multifactorial. Indeed, several factors, including diet particle size [2], management [3], gastric microbiota composition, infection with Helicobacter suis $[4,5]$, and hormonal changes [6] have been hypothesized to be involved. The pathogenesis of porcine gastric ulceration, however, remains largely unknown [1]. What we do know is that, in contrast to other animal species, in pigs gastric ulcers

\footnotetext{
*Correspondence: chloe.dewitte@ugent.be

${ }^{\dagger} \mathrm{A}$. Smet and F. Haesebrouck shared senior authorship

1 Department of Pathology, Bacteriology and Avian Diseases, Faculty

of Veterinary Medicine, Ghent University, Merelbeke, Belgium

Full list of author information is available at the end of the article
}

develop almost exclusively in the Pars oesophagea, a small area around the opening of the œsophagus which does not contain glands. Since this stomach region is not protected by mucus, it is highly susceptible to irritation with for instance hydrochloric acid, produced in the fundic gland zone of the porcine stomach [1]. Chronic insult of the Pars oesophagea results in hyperkeratosis, erosion and finally ulceration.

Pigs are commonly infected with the zoonotic pathogen $H$. suis [1]. This pathogen mainly colonizes the fundic and pyloric gland zone of the porcine stomach, inducing inflammation and a decreased daily weight gain [5]. It has been hypothesized that alterations in hydrochloric acid production in the glandular region of the stomach, associated with chronic $H$. suis infections, may play a role in the pathogenesis of swine gastric ulceration $[1,4,5]$. Hellemans et al. [4] demonstrated a tropism of $H$. suis for the gastric acid producing parietal cells. Histological analysis of the stomach of $\mathrm{H}$. suis-infected pigs at slaughter age, 
has revealed that these bacteria are often found in close vicinity of parietal cells and even inside the canaliculi of these cells [4]. In addition, $H$. suis can cause degenerative changes and necrosis of parietal cells in porcine, human and rodent models of gastric disease [7, 8]. Recent reports indicated that $H$. suis may disturb homeostasis of porcine parietal cells and affect their expression of genes encoding $\mathrm{H}+/ \mathrm{K}+$ ATPase [9]. The latter is an enzyme typically associated with parietal cells and is involved in gastric acid production by these cells. Not only parietal cells, but also gastrin producing G-cells and somatostatin producing D-cells can be altered during $H$. suis infection [10]. Gastrin stimulates and somatostatin suppresses gastric acid production through their association with CCK-B and SST2 receptors on parietal cells, respectively, suggesting that $H$. suis infection may indeed affect gastric acid secretion through different mechanisms.

The main objectives of this study were to obtain further insights in the mechanisms involved in persistence of $H$. suis in the porcine stomach and in its effects on gastric acid secretion and lesion development. This was studied in naturally $H$. suis infected pigs during the acute and chronic phases of infection. Therefore, the mRNA expression of different cytokines, chemokines and markers for gastric acid secretion was studied, the parietal cell, D-cell and G-cell mass was analyzed and the severity of Pars oesophageal lesions was determined in $H$. suis infected and non-infected 2-3 months old pigs, 6-8 months old pigs and adult sows.

\section{Materials and methods}

\section{Sampling of porcine stomachs}

Sixty-eight stomachs of 6-8 months old pigs and 60 stomachs of adult sows (1-3 years old) were collected over a period of 10 months from 2 slaughterhouses in Flanders, Belgium. The pigs originated from different herds. The stomachs of the $6-8$ months old pigs had also been used in a previous study [11]. In addition, stomachs of 34, 2-3 months old pigs were collected from 2 different pig herds (17 samples from each herd). The stomachs were transported immediately to the laboratory and stored at $4{ }^{\circ} \mathrm{C}$ until further examination within $2 \mathrm{~h}$. The stomachs were opened along the greater curvature and rinsed with sterile tap water. Based on the method of Hessing [12], mucosal lesions of the Pars oesophagea were scored as follows: score 0 for normal mucosa, score 1 for mild hyperkeratosis covering less than $50 \%$ of the surface, score 2 for severe hyperkeratosis covering more than $50 \%$ of the surface, score 3 for hyperkeratosis with few erosions, score 4 for hyperkeratosis with several erosions and score 5 for hyperkeratosis with many erosions or ulceration. Using autoclaved tweezers and scalpels, biopsies of 40-50 mg consisting of mucosa and submucosa were taken from the Pars oesophagea as well as from the cardiac, fundic and pyloric gland zone for quantification of $H$. suis DNA by qPCR. In addition, biopsies consisting of mucosa and submucosa were taken from the fundic and pyloric gland zones to determine mRNA expression levels of genes encoding host factors (markers) involved in gastric acid secretion and inflammation. In order to correlate altered markers with gastritis and the number of parietal cells, D-cells and G-cells, biopsies consisting of mucosa, submucosa and tunica muscularis were taken from fundic and pyloric gland zones, fixed in 10\% phosphate-buffered formalin and used for histopathology and immunohistochemistry.

\section{H. suis quantification}

DNA was extracted from the biopsies of each stomach region, using the Isolate II Genomic DNA Kit ${ }^{\circledR}$ (Bioline, Taunton, USA), according to the instructions of the manufacturer. The presence of $H$. suis DNA was determined using a species-specific, real time quantitative (RT)PCR based on the ureA gene [13]. The copy number of the obtained amplicons was calculated and converted to the number of $H$. suis bacteria per mg gastric tissue, by including tenfold dilutions of an external standard consisting of a $1236 \mathrm{bp}$ segment of the ure $A B$ gene cluster from $H$. suis strain HS5 [14].

\section{Histopathology and immunohistochemistry}

The biopsies were embedded in paraffin, sectioned at $5 \mu \mathrm{m}$, rehydrated, deparaffinized, stained with hæmatoxylin and eosin, dehydrated and finally mounted with a coverslip for light microscopic evaluation. The severity of gastritis was scored according to the Updated Sydney System with some modifications [5, 15]. Both diffuse infiltration with inflammatory cells and the presence of lymphoid aggregates and lymphoid follicles in the mucosa and submucosa were taken into consideration. The infiltration of mononuclear and polymorphonuclear cells was scored as follows: score 0 for absence of infiltration, score 1 for mild infiltration, score 2 for moderate infiltration and score 3 for marked infiltration. In addition, the formation of lymphoid follicle formation was scored as follows: score 0 for absence of lymphoid aggregates, score 1 for presence of a small number of lymphoid aggregates $(n<5)$, score 2 for a large number of lymphoid aggregates $(n \geq 5)$ and/or the presence of 1 organized lymphoid follicle and score 3 for the presence of at least 2 organized lymphoid follicles. Based on the scoring of the diffuse infiltration with inflammatory cells and the presence of lymphoid aggregates and lymphoid follicles, an overall gastritis score was obtained. Therefore, the average score was calculated for each $H$. suis-negative and $H$. suis-positive age group and this for the pyloric and fundic 
gland zone. When an overall score of $0 \leq n \leq 1 ; 1<n \leq 2$ or $2<n \leq 3$ was obtained, the gastritis was considered as mild, moderate and severe, respectively.

To determine the number of parietal cells, D-cells and G-cells, 3 consecutive sections of $5 \mu \mathrm{m}$ were cut from the paraffin embedded tissues. After rehydration and deparaffinization, heat-induced antigen retrieval was performed in citrate buffer ( $\mathrm{pH}$ 6.0) using a microwave oven. Slides were incubated with $3 \% \mathrm{H}_{2} \mathrm{O}_{2}$ in methanol (5 min) to block endogenous peroxidase activity and with $30 \%$ goat serum (30 min) to block non-specific reactions. Parietal cells were identified by immunohistochemical staining for the $\mathrm{H}+/ \mathrm{K}+$ ATPase using a mouse monoclonal antibody (1/200; Abcam Ltd, Cambridge, United Kingdom) and a biotinylated goat anti-mouse IgG antibody (1/200; Agilent Technologies, Santa Clara, California, USA) [9]. D-cells and G-cells were identified by immunohistochemical staining using a rabbit polyclonal anti-somatostatin and anti-gastrin antibody, respectively (1/600; Agilent Technologies, Santa Clara, California, USA) and a biotinylated goat anti-rabbit IgG antibody (1/600; Agilent Technologies, Santa Clara, California, USA). After rinsing, the sections were incubated with a streptavidin-biotin-HRP complex (Agilent Technologies, Santa Clara, California, USA) [10]. The color was developed with diaminobenzidine tetrahydrochloride (DAB) and $\mathrm{H}_{2} \mathrm{O}_{2}$. Finally, positive D-cells and G-cells were counted in five randomly chosen high power fields (magnification: $\times 400$ ), both in the fundic and pyloric gland zone. The average number of positive cells per high power field was then calculated for each pig in both stomach regions. As a positive control for the parietal cell staining, the fundic gland zone of a non- $H$. suis infected pig was used, as this zone is known to contain large numbers of these cells [10]. The pyloric gland zone of this pig was used as a positive control for D-cells and G-cells staining. This zone indeed contains large numbers of these cell types [10]. Negative controls to confirm the specificity of the secondary antibodies were obtained by incubating the sections without the primary antibodies. In addition, the cardiac gland zone was also used as a negative control, as this stomach region is known to contain only mucus and bicarbonate producing cells [10].

\section{Expression analysis of markers for inflammation and gastric acid secretion}

RNA was extracted from the gastric biopsies using the RNeasy Mini Kit ${ }^{\circledR}$ (Qiagen, Hilden, Germany) according to the manufacturer's instructions. The obtained RNA concentrations were measured using a NanoDrop ${ }^{\circledR}$ spectrophotometer (Isogen Life Science, Utrecht, The Netherlands), after which the concentration of all samples was adjusted to $1 \mu \mathrm{g} / \mu \mathrm{L}$, followed by cDNA synthesis using the iScript $^{\mathrm{TM}}$ cDNA Synthesis Kit (Bio-Rad, Hercules, California, USA). Expression of genes encoding host factors involved in gastric acid secretion $(\mathrm{H}+/ \mathrm{K}+$ ATPase, Sonic Hedgehog, KCNQ1, gastrin, the cholinergic muscarinic M3 receptor, somatostatin, the histamine $\mathrm{H} 2$ receptor and the gastrin $\mathrm{CCK}-\mathrm{B}$ receptor), mucosal integrity (claudin 18) and inflammation (IL-4, IL-8, IL-10, IL17A, IL-1 $\beta$, IFN- $\gamma$ and CXCL13) was analyzed. HPRT, Cyc5 and ACTB have been shown to have a stable mRNA expression and were therefore included as reference genes [9]. All primer sequences are shown in the Additional file 1 . The mRNA expression levels of the reference and target genes were quantified using RT-qPCR, as described earlier [16]. No-template control reaction mixtures were included and all samples were run in duplicate. The threshold cycle $(\mathrm{Ct})$-values were first normalized to the geometric mean of the Ct-values of the reference genes. Fold changes were calculated using $\Delta \Delta \mathrm{CT}$ method with the means of Ct-values from the $H$. suis negative pigs. Finally, for each target gene, the results were expressed as fold changes of the mRNA expression of $H$. suis positive pigs relative to mRNA expression levels of $H$. suis negative pigs and this for each age group separately.

\section{Statistical analysis}

Statistical analysis was performed using SPSS statistics $24^{\circledR}$ (IBM, New York, USA). Differences in severity of Pars oesophageal lesions, severity of gastritis, number of parietal cells, D-cells and G-cells and fold changes of the markers for gastric acid secretion and inflammation between the $H$. suis-negative and $H$. suis-positive groups were investigated using the non-parametric Kruskal-Wallis $\mathrm{H}$ test with Bonferroni correction. A $p$ value $\leq 0.05$ was considered to be significant. Correlations between mucosal lesions, severity of gastritis, number of parietal cells, D-cells and G-cells, fold changes and the number of $H$. suis bacteria were examined using the Pearson correlation coefficient. Differences were considered statistically significant at $p \leq 0.05$.

\section{Results}

$H$. suis prevalence and association with mucosal lesions, gastritis and number of parietal cells, D-cells and G-cells Two-3 months old pigs

The prevalence of $H$. suis was $47 \%$ and the average number of $H$. suis bacteria per mg tissue was higher in the pyloric gland zone than in the other stomach regions $(p<0.001)$ (Additional file 2). Almost all pigs had H. suis DNA in the fundic and pyloric gland zone $(=15 / 16)$. On gross examination, all pigs showed an intact mucosa or mild hyperkeratosis of the Pars aesophagea (Table 1), with moderate gastritis in the fundic and pyloric gland zone 
Table 1 General overview of the score distribution of lesions (\%) in the Pars œsophagea of pigs of different ages

\begin{tabular}{|c|c|c|c|c|c|c|}
\hline \multirow[t]{2}{*}{ Age group } & \multicolumn{6}{|c|}{ Pars œesophagea-lesion score } \\
\hline & $0(\%)$ & $1(\%)$ & $2(\%)$ & $3(\%)$ & $4(\%)$ & $5(\%)$ \\
\hline $2-3$ months old $(n=34)$ & 38 & 44 & 18 & 0 & 0 & 0 \\
\hline H. suis positive $(n=16)$ & 37 & 44 & 19 & 0 & 0 & 0 \\
\hline H. suis negative $(n=18)$ & 39 & 44 & 17 & 0 & 0 & 0 \\
\hline $6-8$ months old $(n=68)$ & 2 & 27 & 50 & 13 & 3 & 5 \\
\hline H. suis positive $(n=55)$ & 0 & 13 & 61 & 17 & 4 & 6 \\
\hline H. suis negative $(n=13)$ & 8 & 92 & 0 & 0 & 0 & 0 \\
\hline Adult sows $(n=60)$ & 0 & 5 & 20 & 15 & 10 & 50 \\
\hline H. suis positive $(n=55)$ & 0 & 5 & 20 & 13 & 9 & 53 \\
\hline H. suis negative $(n=5)$ & 0 & 0 & 20 & 40 & 20 & 20 \\
\hline
\end{tabular}

Score $0=$ normal mucosa, $1=$ mild hyperkeratosis covering less than $50 \%$ of the surface, $2=$ severe hyperkeratosis covering more than $50 \%$ of the surface, $3=$ hyperkeratosis with few erosions, $4=$ hyperkeratosis with several erosions, $5=$ hyperkeratosis with many erosions or ulceration, $n=$ total number of investigated pigs' stomachs per age group. The data are shown as the percentage of pigs showing a certain lesion score.

(Additional file 3). The scores for Pars oesophageal lesions and gastritis were not significantly different between the $H$. suis-negative and $H$. suis-positive pigs. Similarly, the number of G-cells and D-cells in the pyloric gland zone did not differ between the $H$. suis-negative and $H$. suispositive group (Figures $1 \mathrm{~A}$ and $\mathrm{B}$ ). A small number of G-cells and D-cells was detected in the fundic gland zone of the pigs, varying from 0 to 3 per high power field and independent from the $H$. suis status (data not shown). In the fundic gland zone of both $H$. suis-infected and noninfected pigs, the number of parietal cells was high in each high power field ( $>800 /$ field), making the counting impossible. A small number of parietal cells was detected in the pyloric gland zone of the pigs, varying from 0 to 2 per high power field and independent from the $H$. suis status (data not shown). For the $H$. suis-positive pigs, statistical analysis did not reveal significant correlations between mucosal lesions, gastritis and the number of $H$. suis bacteria. Analysis of gene expression, histopathology and immunohistochemistry was done on samples from all pigs in this age category (see below).

\section{Six-8 months old pigs}

Helicobacter suis was detected in $81 \%$ of the investigated stomachs. The average number of $H$. suis bacteria per mg tissue was similar for the fundic and pyloric gland zone (Additional file 2). H. suis DNA was detected in the fundic and pyloric gland zone of all $H$. suis-infected pigs. Severe hyperkeratosis and erosions were only seen in the $H$. suis-positive group (Table 1 ). The number of

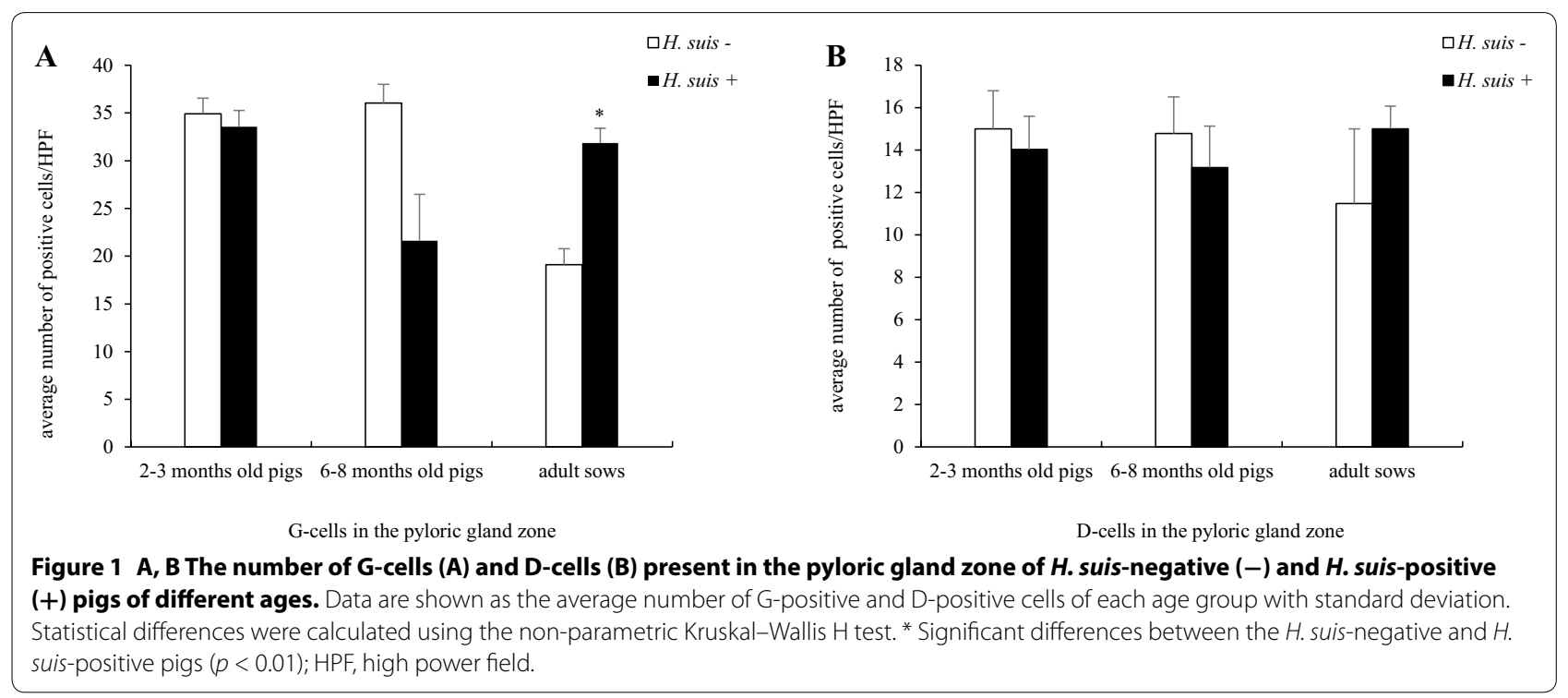


D-cells did not differ between the $H$. suis-negative and $H$. suis-positive group, whereas the number of G-cells was decreased in the $H$. suis-positive group $(p=0.054)$ (Figures $1 \mathrm{~A}$ and $\mathrm{B}$ ). A small number of G-cells and D-cells was detected in the fundic gland zone of the pigs, varying from 0 to 3 per high power field and independent from the $H$. suis status (data not shown). In the fundic gland zone of both $H$. suis-infected and non-infected pigs, the number of parietal cells was high in each high power field ( $>800 /$ field), making the counting impossible. A small number of parietal cells was detected in the pyloric gland zone of the pigs, varying from 0 to 2 per high power field and independent from the $H$. suis status (data not shown). No significant correlations were detected between the number of $H$. suis bacteria and severity of gastritis. All $H$. suis-negative pigs $(n=13), 5$ pigs with $>1000 \mathrm{H}$. suis bacteria/mg tissue in the fundic gland zone, 5 pigs with $>1000 \mathrm{H}$. suis bacteria/mg tissue in the pyloric gland zone and 5 pigs with $<100 \mathrm{H}$. suis bacteria/mg tissue in both the fundic and pyloric gland zone were selected for analysis of gene expression, histopathology and immunohistochemistry.

\section{Adult sows}

Helicobacter suis was detected in the stomach of 55/60 sows $(92 \%)$. In contrast with the other age groups, the average number of $H$. suis bacteria per mg tissue was higher in the fundic gland zone than in the other stomach regions $(p<0.01)$ (Additional file 2). All H. suis-infected sows had $H$. suis DNA in the fundic and pyloric gland zone. Ulceration was mainly found in the H. suis-positive sows, although this was not significantly different from the $H$. suis-negative group (Table 1 ). No significant differences were detected in the severity of gastritis between the $H$. suis-negative and $H$. suis-positive pigs (Additional file 3). A significant positive correlation was found, however, between the number of $H$. suis bacteria per mg gastric tissue and lymphoid infiltration in the fundic gland zone $(p<0.001)$. The number of $\mathrm{D}$-cells did not differ between the $H$. suis-negative and $H$. suis-positive group, while the number of G-cells was increased in the $H$. suis-positive group $(p=0.002)$ (Figures $1 \mathrm{~A}$ and $\mathrm{B}$; Additional file 4). A small number of G-cells and D-cells was detected in the fundic gland zone of the pigs, varying from 0 to 3 per high power field and independent from the $H$. suis status (data not shown). In the fundic gland zone of both $H$. suis-infected and non-infected pigs, the number of parietal cells was high in each high power field (> 800/field), making the counting impossible. A small number of parietal cells was detected in the pyloric gland zone of the pigs, varying from 0 to 2 per high power field and independent from the $\mathrm{H}$. suis status (data not shown). All $H$. suis-negative sows $(n=5)$ were selected for gene expression, histopathological and immunohistochemical analysis. From the $H$. suis-positive group, these analyses were performed on 20 stomachs, selected as follows: 5 sows with $>1000 \mathrm{H}$. suis bacteria/mg tissue in both the fundic and pyloric gland zone, 5 sows with $>1000 \mathrm{H}$. suis bacteria/mg tissue in the fundic gland zone, 5 sows with $>1000 \mathrm{H}$. suis bacteria/mg tissue in the pyloric gland zone and 5 sows with $<100 \mathrm{H}$. suis bacte$\mathrm{ria} / \mathrm{mg}$ tissue in both the fundic and pyloric gland zone.

\section{Comparison of the different age groups}

The scores given for Pars oesophageal lesions were significantly different between each age group $(p<0.001)$, with more severe lesions in adult sows, followed by 6-8 months old pigs. In contrast, the scores for lymphoid infiltration and lymphoid follicle formation did not differ significantly between the age groups, nor did the number of parietal cells, G-cells and D-cells (Figures 1A and B; Additional file 3). Although the prevalence of $H$. suis progressively increased with age, the number of $H$. suis bacteria per mg tissue decreased with age, especially in the pyloric gland zone $(p<0.05$; Figure 2$)$. Significantly higher scores for lymphoid infiltration and lymphoid follicle formation were found in the pyloric gland zone compared to the fundic gland zone $(p<0.005)$, independent from the $H$. suis status, and this in all age groups.

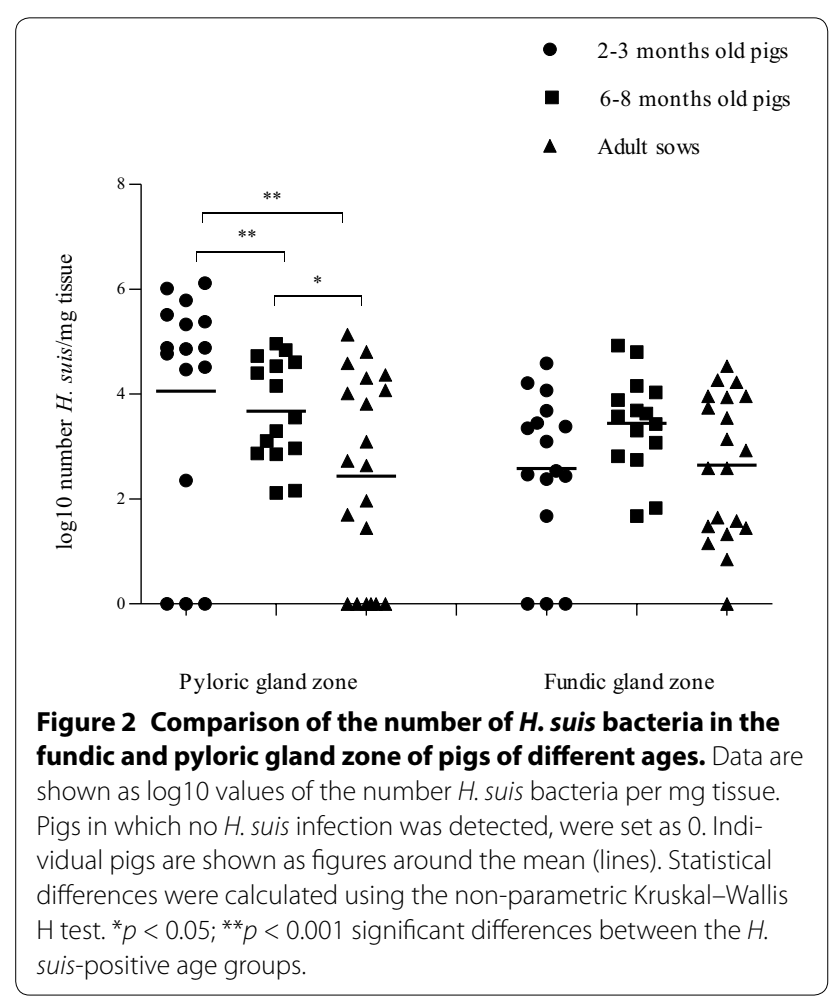


Gene expression analysis of markers for inflammation

\section{Two-3 months old pigs}

Compared to the non-infected group, the mRNA expression of CXCL13 was significantly upregulated in the fundic and pyloric gland zones of the $H$. suis-infected group
( $p=0.027$ and $<0.001$, respectively), as well as the IL-8 and IL-1 $\beta$ transcript levels in the pyloric gland zone $(p=0.001$ and 0.034 , respectively). In contrast, IL-17A was significantly downregulated in the pyloric gland zone $(p=0.039)$ (Figures 3A and B; Additional file 5). Since

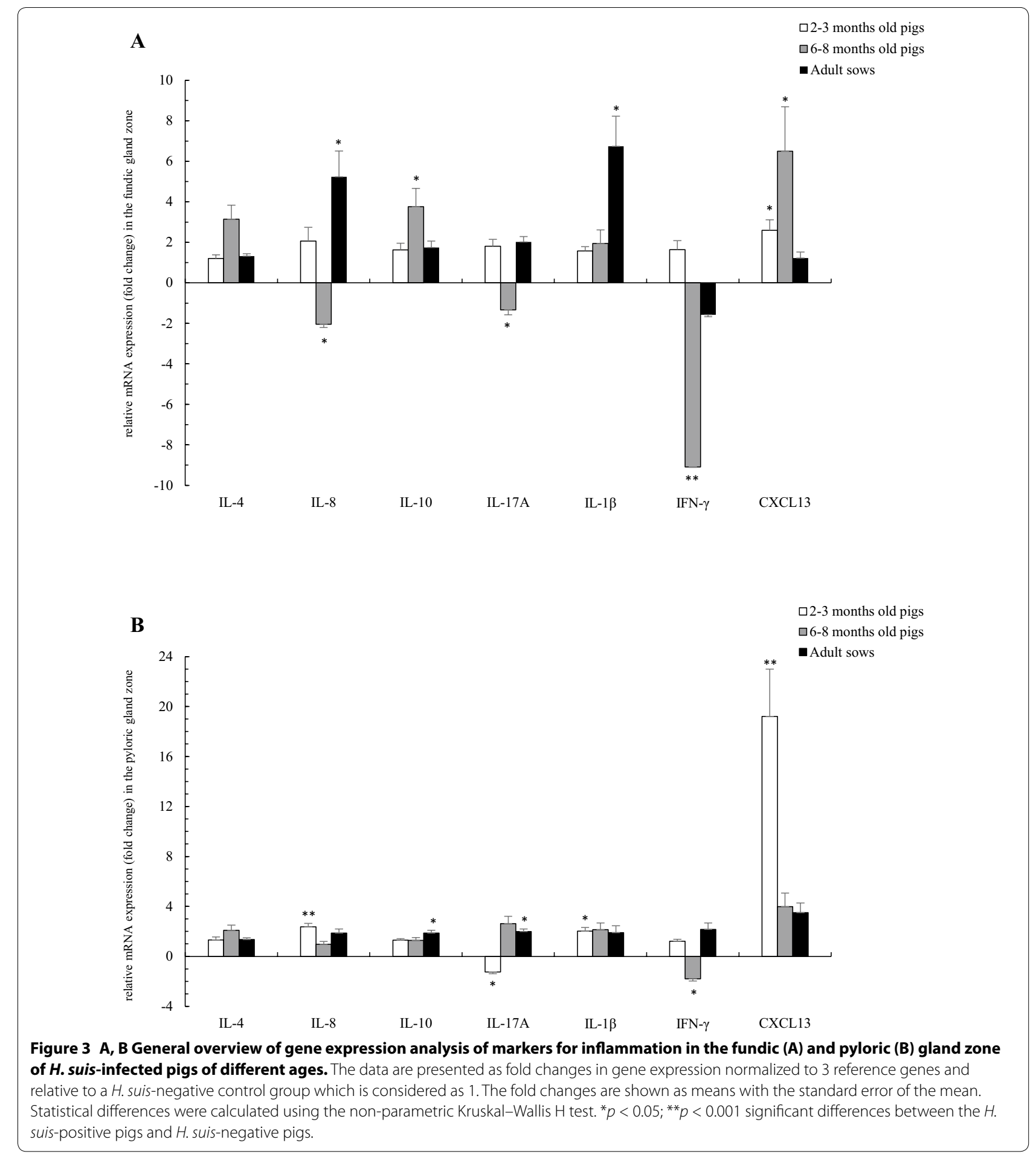


significant correlations were found between both, the altered fold changes of IL-8, IL-17A, IL-1 $\beta$ and CXCL13 were more pronounced in pigs with a high number of $H$. suis bacteria per mg gastric tissue (Additional file 6).

\section{Six-8 months old pigs}

Upregulated expression of inflammatory cytokines was as described in the study of Bosschem et al. [11]. In brief, compared to the non-infected group, in the $H$. suis-positive group, significant upregulations of IL-10 and CXCL13 were detected in the fundic gland zone ( $p=0.047$ and 0.011 , respectively). The expressions of IL-4 in the fundic gland zone and that of IL-4, IL-17A and CXCL13 in the pyloric gland zone were also upregulated, although not significantly. A significant downregulation of IL-8 and IL-17A was detected in the fundic gland zone ( $p=0.040$ and 0.029 , respectively), while IFN- $\gamma$ was significantly downregulated in both fundic and pyloric gland zone ( $p<0.001$ and $=0.005$, respectively) (Figures $3 \mathrm{~A}$ and B; Additional file 5). Since significant correlations were found between both, the altered fold changes of IL-4, IL-8, IL-10, IL-17A, IFN- $\gamma$ and CXCL13 were more pronounced in pigs with $>1000 \mathrm{H}$. suis bacteria/mg gastric tissue (Additional file 6).

\section{Adult sows}

Compared to the non-infected sows, in the $H$. suisinfected adult sows, the mRNA expression of IL- 8 and IL- $1 \beta$ was significantly upregulated in the fundic gland zone ( $p=0.018$ for IL- 8 and 0.037 for IL- $1 \beta)$, while IL-10 and IL-17A were upregulated in the pyloric gland zone of $H$. suis-infected sows $(p=0.019$ and 0.042 , respectively). Although not significantly, increased IL-10 and IL-17A mRNA expression was also detected in the fundic gland zone and in the pyloric gland zone for IFN- $\gamma$ and CXCL13 (Figures 3A and B; Additional file 5). Since significant correlations were found between both, the altered fold changes of IL-8, IL-10, IL-17A, IL-1 $\beta$, IFN- $\gamma$ and CXCL13 were more distinct in pigs with $>1000 \mathrm{H}$. suis bacteria/mg gastric tissue (Additional file 6).

\section{Gene expression analysis of markers for gastric acid secretion \\ Two-3 months old pigs}

Compared to the non-infected group, in the $H$. suisinfected 2-3 months old pigs, the majority of the markers for gastric acid secretion were not altered, except for a significant downregulated expression of the M3-receptor in the pyloric gland zone $(p=0.027)$. The expression of KCNQ1 was upregulated in the fundic gland zone, while somatostatin was downregulated in the pyloric gland zone, although not significantly (Figures 4A and B; Additional file 7). Since significant correlations were found between both, the altered fold changes of KCNQ1, M3-receptor and somatostatin were more pronounced in pigs with $>1000 \mathrm{H}$. suis bacteria/mg per mg gastric tissue (Additional file 8). In addition, since significant correlations were found between both markers, the altered fold change of somatostatin was more pronounced in pigs with a high expression of CXCL13 and IL-1 $\beta$ (Additional file 9).

\section{Six-8 months old pigs}

Compared to the non-infected group, in the $H$. suisinfected group claudin 18, gastrin, M3 receptor and CCK-B receptor were significantly downregulated in the fundic gland zone $(p=0.022,0.040,0.002$ and 0.004, respectively), whereas Sonic Hedgehog and somatostatin mRNA levels were significantly upregulated in the pyloric gland zone ( $p=0.048$ and 0.007 , respectively). The $\mathrm{H}+/ \mathrm{K}+$ ATPase expression in the pyloric gland zone was upregulated as well, although not significantly. In the pyloric gland zone of $9 \mathrm{H}$. suis-infected pigs, the expression of gastrin was upregulated (Figures 4A and B; Additional file 7), while for $6 \mathrm{H}$. suis-infected pigs the expression was not altered. Since significant correlations were found between both, the altered fold changes of $\mathrm{H}+/ \mathrm{K}+$ ATPase, Sonic Hedgehog, claudin 18, gastrin, M3 receptor, somatostatin and CCK-B receptor were more pronounced in pigs with $>1000 \mathrm{H}$. suis bacteria/mg gastric tissue (Additional file 8). In addition, since significant correlations were found between both markers, the altered fold changes of claudin 18, M3 receptor, somatostatin and CCK-B receptor were more pronounced in pigs with lower expressions of IL-8, IL-17A and IFN- $\gamma$ (Additional file 9).

\section{Adult sows}

Compared to the non-infected sows, most markers for gastric acid secretion were upregulated in the $H$. suisinfected sows. The expression of genes encoding $\mathrm{H}+$ / $\mathrm{K}+\mathrm{ATPase}$, claudin 18, $\mathrm{H} 2$ receptor and CCK-B receptor were significantly upregulated in the fundic and pyloric gland zone of the $H$. suis-infected sows ( $p=0.049$, $0.002,0.019,0.049,0.002,0.012,0.015$ and 0.012 , respectively). In addition, KCNQ1 and gastrin transcript levels were significantly upregulated in the fundic gland zone ( $p=0.012$ and $<0.001$, respectively), whereas a significant downregulated mRNA expression of the M3 receptor in the pyloric gland zone was noticed ( $p=0.049$ ). An upregulated expression of Sonic Hedgehog was also detected in the fundic and pyloric gland zone, although not significant (Figures 4A and B; Additional file 7). Since significant correlations were found between both, the altered fold changes of genes encoding $\mathrm{H}+/ \mathrm{K}+$ ATPase, Sonic Hedgehog, claudin 18, KCNQ1 and CCK-B 

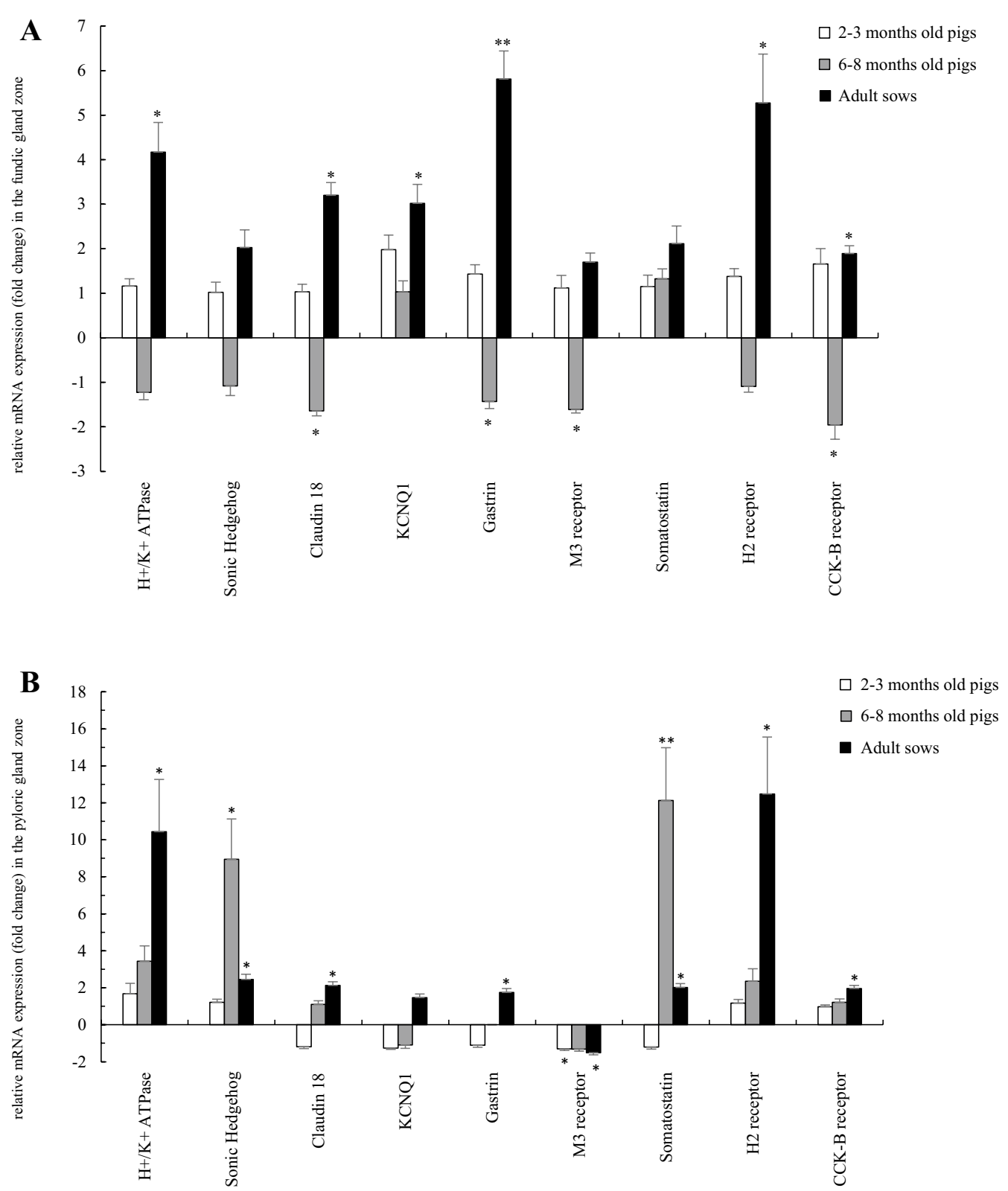

Figure 4 A, B General overview of gene expression analysis of markers for gastric acid secretion in the fundic (A) and pyloric (B) gland zone of $\boldsymbol{H}$. suis-infected pigs of different ages. The data are presented as fold changes in gene expression normalized to 3 reference genes and relative to a H. suis-negative control group which is considered as 1. The fold changes are shown as means with the standard error of the mean. The average fold change of gastrin in the pyloric gland zone of 6-8 months old pigs is not shown, since these values were too high ( $231.97 \pm 64.63)$. Statistical differences were calculated using the non-parametric Kruskal-Wallis H test. ${ }^{*} p<0.05 ;{ }^{* *} p<0.001$ significant differences between the H. suis-positive pigs and H. suis-negative pigs.

receptor were more pronounced in pigs with $>1000 \mathrm{H}$. suis bacteria/mg gastric tissue (Additional file 8 ). In addition, since significant correlations were found between both markers, the altered fold changes of genes encoding $\mathrm{H}+/ \mathrm{K}+$ ATPase, Sonic Hedgehog, claudin 18, gastrin and CCK-B receptor were more pronounced in pigs with 
high expressions of IL-10, IL-17A and IFN- $\gamma$ (Additional file 9). Furthermore, since significant positive correlations were found between both, an increased G-cell number may have contributed to the increased expression of gastrin in both fundic and pyloric gland zone $(r=0.562$, $p=0.003 ; r=0.465, p=0.022$, respectively).

\section{Discussion}

In the present study, the prevalence of $H$. suis was $47 \%$ in $2-3$ months old pigs and increased to $81 \%$ in pigs at slaughter age, which is in line with the results of previous studies [17-19].

The prevalence of $H$. suis was very high in adult animals, indicating that the host immune response is not able to clear the infection. In a recent study, Bosschem et al. [11] showed that $H$. suis induces a semimaturation of porcine monocyte-derived dendritic cells, characterized by increased expression of CD25, CD80/86 and CD40, but impaired expression of MHC class II molecules on the surface of these cells. It was suggested that this impaired dendritic cell response may elicit the expansion of Treg cells, which may help to establish a chronic infection as Treg cells are immune-suppressive and tolerogenic [20]. Indeed, a tolerogenic immune response was indicated to be present in this study, since the Treg cell-associated cytokine IL-10 was upregulated in both $H$. suis-infected 6-8 months old pigs and adult sows. In addition, the downregulated expressions of IL-8, IL-17A and especially IFN- $\gamma$ indicated presence of an immune-suppressive environment in the $H$. suisinfected 6-8 months old pigs, which may have contributed to the establishment of a chronic infection. In the $H$. suis-infected adult sows, however, the mRNA expression of the Th17 cell-associated IL-17A was upregulated. We also found a shift in colonization of $H$. suis from the pyloric gland zone during the more acute phase of the infection (2-3 months old pigs) to the fundic gland zone in the more chronic phase of the infection (adults sows) in combination with upregulated expressions of IL-8 and IL-1 $\beta$. Taken together, these findings suggest that shortly after colonization, the immune response is suboptimal, contributing to the persistence of $H$. suis infection. Later a more pronounced immune response is seen, which may result in lower numbers of $H$. suis bacteria in that stomach region. Indeed, although the prevalence of $H$. suis was highest in adult sows, the average number of $H$. suis bacteria per mg tissue decreased with age, as was also observed in other studies [8]. The presence of a specific Treg/Th17 response should be confirmed in future studies, where the expansion of Treg and Th17 cells is directly assessed by the use of staining or flow cytometry.

Interestingly, the expression of CXCL13 was upregulated in $H$. suis-infected pigs of each age group. Since this chemokine attracts B-lymphocytes, its upregulation may be important for the development of a specific local immune response towards $H$. suis, but this requires further research [11]. The upregulation of CXCL13 has also been demonstrated in $H$. suis infected mice [21] and has been linked with the development of mucosa associated lymphoid tissue (MALT)-lymphomas in Helicobacter sp. infected human patients [21]. MALT-lymphoma lesions were not detected in the present study and, as far as we know, have not been described in pigs.

In 2-3 months old pigs, the average number of $H$. suis bacteria was the highest in the pyloric gland zone, indicating that $H$. suis colonization starts in this stomach region, as already suggested by others [22]. In adult sows, the average number of $H$. suis bacteria was the highest in the fundic gland zone, indicating a shift in colonization to that region in animals infected during longer periods of time, which is similar to the findings of Hellemans et al. [22]. It appears that when $H$. suis colonizes the stomach epithelium, it triggers an innate immune response in that region, characterized by upregulated expression of the pro-inflammatory cytokines IL- 8 and IL- $1 \beta$ in the pyloric gland zone of $2-3$ months old pigs and in the fundic gland zone of adult sows.

Severe hyperkeratosis and erosions were only seen in the $H$. suis infected 6-8 months old pigs and not in noninfected pigs of this age group. In adult sows, ulceration was also mainly found in the $H$. suis-positive animals, although this was not significantly different from the $H$. suis-negative group, which may be due to the low number of non-infected sows available. In this field study, interpretation of results is further complicated by variation between herds of other factors that may play a role in development of gastric pathologies such as diet, feeding strategy and management [1]. Nevertheless, our findings provide further evidence that $H$. suis may be one of the factors playing a role in the pathogenesis of gastric ulceration in pigs. A similar conclusion was drawn from results of an experimental infection study in pigs [5]. Interestingly, severe lesions in the Pars oesophagea were more frequently found in adult sows compared to 
the other age groups, indicating that ulceration is a longterm process which may affect the majority of the adult pigs.

It is not yet clear how exactly $H$. suis might influence ulcer development in the Pars oesophagea, but alterations in gastric acid secretion might be involved. No clear effects on the markers for gastric acid secretion or number of parietal cells, D-cells and G-cells, and no lesions in the Pars oesophagea were detected in the $H$. suisinfected 2-3 months old pigs (acute phase of infection). In a later phase of infection (6-8 months $H$. suis infected pigs), markers for gastric acid secretion were downregulated, genes encoding somatostatin were upregulated and the number of G-cells was decreased, indicating inhibition of gastric acid secretion. In this age group, lesions in the Pars oesophagea were present in several animals. The prevalence of severe lesions was extremely high in $H$. suis infected adult sows (chronic phase of infection). Markers for gastric acid secretion were upregulated and the number of G-cells was increased in this age group, indicating increased gastric acid secretion. We hypothesize that decreased gastric acid secretion in the glandular part of the stomach may affect the composition of the Pars oesophageal microbiota which may affect development of lesions in this non-glandular part of the stomach. Indeed, higher numbers of a recently described Fusobacterium species, designated $F$. gastrosuis, were detected in the Pars cesophagea of $H$. suis-infected 6-8 months old pigs than in non-infected pigs of the same age group [23]. Increased production of gastric acid during the chronic phase of infection might further aggravate severity of lesions in this stomach region, which is not protected by mucus. Further studies in which for instance the gastric microbiota and $\mathrm{pH}$ are determined in $H$. suis-infected and non-infected pigs, are necessary to confirm or reject this hypothesis.

Several mechanisms might be involved in altered gastric acid secretion in $H$. suis infected animals. A clear parietal cell loss, as described in H. suis-infected Mongolian gerbils and mice $[9,24]$, was not seen in the $H$. suis-infected pigs, although small differences in the number of these cells between the infected and noninfected animals cannot be excluded since counting was impossible in the fundic gland zone. The expression of genes encoding $\mathrm{H}+/ \mathrm{K}+$ ATPase was, however, altered. As this enzyme is typically associated with gastric acid production by parietal cells, this shows that the function of these cells was affected. This is also indicated by altered expression of H2-, M3- and CCK-B receptors, although these receptors are also found on enterochromaffin cells, which were not studied here. The exact mechanism behind the effect of a $H$. suis infection on gastric acid secretion by parietal cells is not clear and requires further studies. Since $H$. suis is often found in close proximity to these host cells, a direct effect of this bacterium on the parietal cells might be involved. Indirect effects probably also play a role since the number of G-cells and/or the expression of gastrin was decreased or enhanced in pigs with downregulated and upregulated expression of $\mathrm{H}+/ \mathrm{K}+$ ATPase, respectively. In $H$. pylori infections, increased gastric acid secretion has been associated with increased expression of genes encoding IL-8 and IL-1 $\beta$ [25-29]. Expression of genes encoding these cytokines was upregulated in the fundic gland zone of adult sows with upregulated expression of genes encoding $\mathrm{H}+/ \mathrm{K}+$ ATPase. Literature dealing with the effect of IL- $1 \beta$ is, however, controversial as Beales and Calam [30] demonstrated that IL-1 $\beta$ inhibits acid secretion in cultured parietal cells.

In $H$. suis infected 6-8 months old pigs and adult sows, expression of genes encoding Sonic Hedgehog was upregulated in the fundic and pyloric gland zone. Since Sonic Hedgehog is involved in the regeneration of damaged epithelium [31], this may indicate a compensation for epithelial loss induced by $H$. suis in these gastric regions. Disruption of the gastric epithelium, followed by regeneration was further suggested by the downregulated expression of genes encoding claudin 18, an important tight junction protein of the stomach [32], in H. suis-infected 6-8 months old pigs and its upregulation in adult sows. The increased IL-10 and IL-17A transcript levels in $H$. suis-infected adult sows may have promoted the regeneration of the gastric epithelium as well, as both cytokines are associated with intestinal barrier restoration [33].

To summarize, we revealed an increased prevalence of $H$. suis and a shift of colonization towards the fundic gland zone in adult sows, while the number of $H$. suis bacteria per mg tissue decreased with age. Gastric erosion and ulceration were more frequently detected in $H$. suis-infected pigs. During the more acute phase of the infection, an innate immune response was indicated to be present, followed by a Treg and Th17 response in pigs colonized during longer periods of time. While no clear alterations in the markers for gastric acid secretion were detected in $2-3$ months old pigs, a decrease and increase were found in 6-8 months old pigs and adult sows, respectively. These results indicate that $H$. suis affects the expression of markers for gastric acid secretion and inflammation and indicate that these effects 


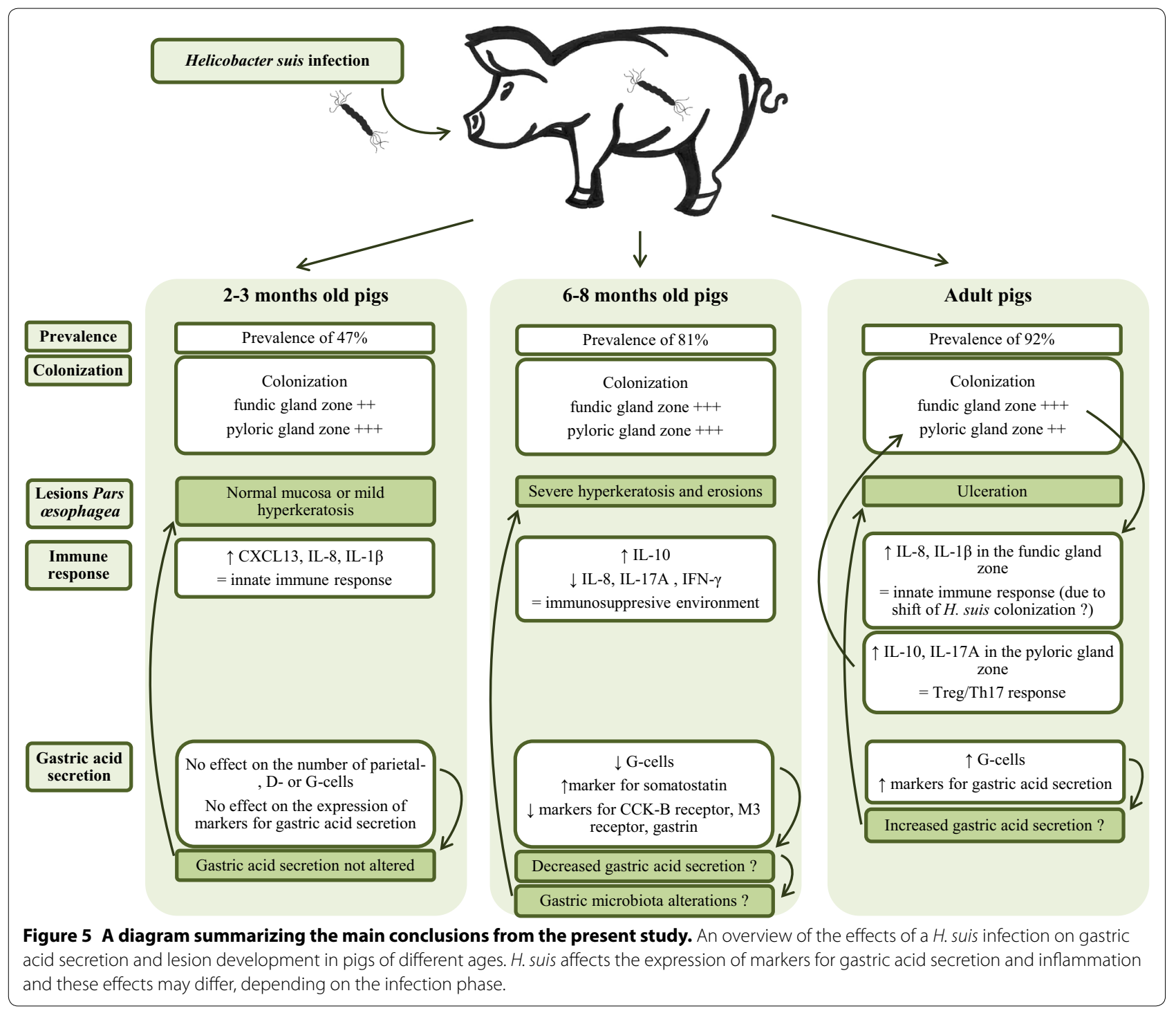

differ, depending on the infection phase. An overview of the main results and conclusions of the present study is presented in Figure 5.

\section{Additional files}

\section{Additional file 1. List of primers used in quantitative RT-PCR for gene expression analysis of markers for gastric acid secretion and inflammation. \\ Additional file 2. The number of $H$. suis bacteria in the different stomach regions of 2-3 months old pigs (A), 6-8 months old pigs (B) and adult sows (C). Data are shown as $\log 10$ values of the average number of $H$. suis bacteria per mg tissue with standard deviation. Statisti- cal differences were calculated using the non-parametric Kruskal-Wallis $\mathrm{H}$ test. ${ }^{*}, p<0.01 ;{ }^{*}, p<0.001$ significant differences between the stomach regions.}

Additional file 3. General overview of the average scores of infiltration with inflammatory cells and lymphoid follicle formation in the fundic and pyloric gland zone of pigs of different ages. Gastritis was scored based on infiltration with inflammatory cells/lymphoid follicle formation, with score $0=$ absence of infiltration/absence of lymphoid aggregates, $1=$ mild infiltration/small number of lymphoid aggregates $(n<5), 2=$ moderate infiltration/large number of lymphoid aggregates $(n>5)$ or presence of 1 organized lymphoid follicle, $3=$ marked infiltration/at least 2 organized lymphoid follicles, $n=$ total number of investigated pigs' stomachs per age group. The data are shown as the average of the administered scores with standard deviation.

Additional file 4. Microscopic visualization of the parietal cells (A), D-cells (B) and G-cells (C-D) in the porcine stomach using immunohistochemistry. (A) $\mathrm{H}+/ \mathrm{K}+$ ATPase staining of the fundic gland zone of a H. suis-positive adult sow, showing parietal cells (brown). No clear parietal cell loss was detected. Original magnification $\times 100$. (B) Somatostatin staining of the pyloric gland zone of a H. suis-positive adult sow, showing D-cells (brown). Original magnification $\times 200$. (C) Gastrin staining of the pyloric gland zone of a H. suis-positive adult sow, showing G-cells (brown). Original magnification $\times 200$. (D) Gastrin staining of the pyloric gland zone of a H. suis-negative sow, showing G-cells (brown). Original magnification $\times 200$. The number of $\mathrm{G}$-cells in the H. suis-negative sow (D) is lower than observed in the H. suis-positive sow (C). 
Additional file 5. Overview of the relative fold changes of altered markers for inflammation in $\boldsymbol{H}$. suis infected pigs of different ages. The data are presented as fold changes in gene expression normalized to 3 reference genes and relative to the $H$. suis-negative control group which is considered as 1 . The fold changes are shown as means with the standard error of the mean. Statistical differences were calculated using the non-parametric Kruskal-Wallis $\mathrm{H}$ test. A p value lower than 0.05 is considered to be significant.

Additional file 6. Overview of important correlations between markers for inflammation and the number of $\boldsymbol{H}$. suis bacteria in pigs of different ages. $r$ = Pearson correlation coefficient, calculated using SPSS Statistics $24^{\circledR}$. A r-value close to 1 indicates a strong, positive correlation, whereas a $r$-value of -1 indicates a strong, negative correlation. A p-value lower than 0.05 is considered to be significant.

Additional file 7. Overview of relative fold changes of altered markers for gastric acid secretion in $\mathbf{H}$. suis-infected pigs of dif-

ferent ages. The data are presented as fold changes in gene expression normalized to 3 reference genes and relative to theH. suis-negative control group which is considered as 1 . The fold changes are shown as means with the standard error of the mean. Statistical differences were calculated using the non-parametric Kruskal-Wallis H test. A p-value lower than 0.05 is considered to be significant.

Additional file 8. Overview of important correlations between markers for gastric acid secretion and the number of $H$. suis bacteria in pigs of different ages. $r=$ Pearson correlation coefficient, calculated using SPSS Statistics $24^{\circledR}$. A r-value close to 1 indicates a strong, positive correlation, whereas a $r$-value of -1 indicates a strong, negative correlation. $P$-values lower than 0.05 are considered to be significant.

Additional file 9. Correlations of altered markers for gastric acid secretion with the number of $H$. suis bacteria and with the altered markers for inflammation in $\mathbf{H}$. suis-infected pigs of different age groups. $r=$ Pearson correlation coefficient, calculated using SPSS Statistics $24^{\circledR}$. A r-value close to 1 indicates a strong, positive correlation, whereas a $r$-value of -1 indicates a strong, negative correlation. $P$-values lower than 0.05 are considered to be significant./= no clear correlation, yes $=$ correlation with $H$. suis colonization rate (see Additional files 6,8 for the $r$ and $p$-values)

\section{Competing interests}

The authors declare that they have no competing interests.

\section{Authors' contributions}

$\mathrm{CDW}, \mathrm{FH}$ and $\mathrm{BF}$ participated in the design of the study. CDW carried out the experiments, analyzed the data and drafted the manuscript. FH, AS and BF coordinated the study and participated in the design of the study, analysis of the data and drafting of the manuscript. All authors read and approved the final manuscript.

\section{Acknowledgements}

This work was supported by a grant from the Special Research Fund of Ghent University (BOF), Ghent, Belgium (Grant No. 01D20414). We thank Marjan Steppe and Christian Puttevils for their excellent technical support with the tissue staining.

\section{Author details}

1 Department of Pathology, Bacteriology and Avian Diseases, Faculty of Veterinary Medicine, Ghent University, Merelbeke, Belgium. ${ }^{2}$ Department of Virology, Parasitology, Immunology, Faculty of Veterinary Medicine, Ghent University, Merelbeke, Belgium. ${ }^{3}$ Laboratory of Experimental Medicine and Pediatrics, Faculty of Medicine and Health Sciences, Antwerp University, Antwerp, Belgium.

\section{Publisher's Note}

Springer Nature remains neutral with regard to jurisdictional claims in published maps and institutional affiliations.
Received: 22 March 2017 Accepted: 31 May 2017

Published online: 15 June 2017

\section{References}

1. Haesebrouck F, Pasmans F, Flahou B, Chiers K, Baele M, Meyns T, Decostere A, Ducatelle R (2009) Gastric helicobacters in domestic animals and nonhuman primates and their significance for human health. Clin Microbiol Rev 22:202-223

2. Ayles HL, Friendship RM, Ball RO (1996) Effect of dietary particle size on gastric ulcers, assessed by endoscopic examination, and relationship between ulcer severity and growth performance of individually fed pigs. Swine Health Prod 4:211-216

3. Herskin MS, Jensen HE, Jespersen A, Forkman B, Jensen MB, Canibe N, Pedersen $L J$ (2016) Impact of the amount of straw provided to pigs kept in intensive production conditions on the occurrence and severity of gastric ulceration at slaughter. Res Vet Sci 104:200-206

4. Hellemans A, Chiers K, Decostere A, De Bock M, Haesebrouck F, Ducatelle R (2007) Experimental infection of pigs with "Candidatus Helicobacter suis". Vet Res Commun 31:385-395

5. De Bruyne E, Flahou B, Chiers K, Meyns T, Kumar S, Vermoote M, Pasmans F, Millet S, Dewulf J, Haesebrouck F, Ducatelle R (2012) An experimental Helicobacter suis infection causes gastritis and reduced daily weight gain in pigs. Vet Microbiol 160:449-454

6. Bubenik GA, Ayles HL, Friendship RM, Brown GM, Ball RO (1998) Relationship between melatonin levels in plasma and gastrointestinal tissues and the incidence and severity of gastric ulcers in pigs. J Pineal Res 24:62-66

7. Joo M, Ji EK, Sun HC, Kim H, Chi JG, Kim KA, Jeon HY, June SL, Moon YS, Kim KM (2007) Helicobacter heilmannii-associated gastritis: clinicopathologic findings and comparison with Helicobacter pylori-associated gastritis. J Korean Med Sci 22:63-69

8. Flahou B, Haesebrouck F, Pasmans F, D'Herde K, Driessen A, Van Deun K, Smet A, Duchateau L, Chiers K, Ducatelle R (2010) Helicobacter suis causes severe gastric pathology in mouse and mongolian gerbil models of human gastric disease. PLoS One 5:e14083

9. Zhang G, Ducatelle R, Mihi B, Smet A, Flahou B, Haesebrouck F (2016) Helicobacter suis affects the health and function of porcine gastric parietal cells. Vet Res 47:101

10. Sapierzyński R, Fabisiak M, Kizerwetter-Swida M, Cywińska A (2007) Effect of Helicobacter sp. infection on the number of antral gastric endocrine cells in swine. Pol J Vet Sci 10:65-70

11. Bosschem I, Flahou B, Van Deun K, De Koker S, Volf J, Smet A, Ducatelle R, Devriendt B, Haesebrouck F (2017) Species-specific immunity to Helicobacter suis. Helicobacter. doi:10.1111/hel.12375

12. Hessing MJC, Geudeke MJ, Scheepens CJM, Tielen MJM, Schouten WGP, Wiepkema PR (1992) Mucosal lesion sin the Pars oesophagea in pigs prevalence and influence of stress. Tijdschr Diergeneeskd 117:445-450

13. Blaecher C, Smet A, Flahou B, Pasmans F, Ducatelle R, Taylor D, Weller C, Bjarnason I, Charlett A, Lawson AJ, Dobbs RJ, Dobbs SM, Haesebrouck F (2013) Significantly higher frequency of Helicobacter suis in patients with idiopathic parkinsonism than in control patients. Aliment Pharmacol Ther 38:1347-1353

14. O'Rourke JL, Solnick JV, Neilan BA, Seidel K, Hayter R, Hansen LM, Lee A (2004) Description of "Candidatus Helicobacter heilmannii" based on DNA sequence analysis of $16 S$ rRNA and urease genes. Int J Syst Evol Microbiol 54:2203-2211

15. Dixon MF, Genta RM, Yardley JH, Correa P (1996) Classification and grading of gastritis. The updated Sydney system. International workshop on the histopathology of gastritis, Houston 1994. Am J Surg Pathol 20:1161-1181

16. Flahou B, Deun KV, Pasmans F, Smet A, Volf J, Rychlik I, Ducatelle R, Haesebrouck $F$ (2012) The local immune response of mice after Helicobacter suis infection: strain differences and distinction with Helicobacter pylori. Vet Res 43:1

17. Roosendaal R, Vos JH, Roumen T, van Vugt R, Cattoli G, Bart A, Klaasen HL, Kuipers EJ, Vandenbroucke-Grauls CM, Kusters JG (2000) Slaughter pigs are commonly infected by closely related but distinct gastric ulcerative lesion-inducing gastrospirilla. J Clin Microbiol 38:2661-2664 
18. Choi YK, Han JH, Joo HS (2001) Identification of novel Helicobacter species in pig stomachs by PCR and partial sequencing. J Clin Microbiol 39:3311-3315

19. Park JH, Seok SH, Cho SA, Baek MW, Lee HY, Kim DJ, Park JH (2004) The high prevalence of Helicobacter sp. in porcine pyloric mucosa and its histopathological and molecular characteristics. Vet Microbiol 104:219-225

20. Kao JY, Zhang M, Miller MJ, Mills JC, Wang B, Liu M, Eaton KA, Zou W, Berndt BE, Cole TS, Takeuchi T, Owyang SY, Luther J (2010) Helicobacter pylori immune escape is mediated by dendritic cell-induced treg skewing and Th17 suppression in mice. Gastroenterology 138:1046-1054

21. Zhang G, Ducatelle R, De Bruyne E, Joosten M, Bosschem I, Smet A, Haesebrouck F, Flahou B (2015) Role of $\gamma$-glutamyltranspeptidase in the pathogenesis of Helicobacter suis and Helicobacter pylori infections. Vet Res 46:31

22. Hellemans A, Chiers K, De Bock M, Decostere A, Haesebrouck F, Ducatelle R, Maes D (2007) Prevalence of "Candidatus Helicobacter suis" in pigs of different ages. Vet Rec 161:189-192

23. De Witte C, Flahou B, Ducatelle R, Smet A, De Bruyne E, Cnockaert M, Taminiau B, Daube G, Vandamme P, Haesebrouck F (2017) Detection, isolation and characterization of Fusobacterium gastrosuis sp. nov. colonizing the stomach of pigs. Syst Appl Microbiol 40:42-50

24. Joosten M, Blaecher C, Flahou B, Ducatelle R, Haesebrouck F, Smet A (2013) Diversity in bacterium-host interactions within the species Helicobacter heilmannii sensu stricto. Vet Res 44:65

25. Calam J (1999) Helicobacter pylori modulation of gastric acid. Yale J Biol Med 72:195-202
26. Calam J (1996) Helicobacter pylori and hormones. Yale J Biol Med 69:39-49

27. Haruma K, Kawaguchi H, Kohmoto K, Okamoto S, Yoshihara M, Sumii K, Kajiyama G (1995) Helicobacter pylori infection, serum gastrin, and gastric acid secretion in teen-age subjects with duodenal ulcer, gastritis, or normal mucosa. Scand J Gastroenterol 30:322-326

28. Lehmann FS, Golodner EH, Wang J, Chen MC, Avedian D, Calam J, Walsh $\mathrm{H}$, Dubinett S, Soll AH (1996) Mononuclear cells and cytokines stimulate gastrin release from canine antral cells in primary culture. Am J Physiol 270:G783-G788

29. Beales I, Calam J, Post L, Srinivasan S, Yamada T, DelValle J (1997) Effect of transforming growth factor alpha and interleukin 8 on somatostatin release from canine fundic D cells. Gastroenterology 112:136-143

30. Beales IL, Calam J (1998) Interleukin 1 beta and tumour necrosis factor alpha inhibit acid secretion in cultured rabbit parietal cells by multiple pathways. Gut 42:227-234

31. Feng R, Xiao C, Zavros Y (2012) The role of Sonic Hedgehog as a regulator of gastric function and differentiation. Vitam Horm 88:473-489

32. Caron TJ, Scott KE, Fox JG, Hagen SJ (2015) Tight junction disruption: Helicobacter pylori and dysregulation of the gastric mucosal barrier. World J Gastroenterol 21:11411-11427

33. Lee SH (2015) Intestinal permeability regulation by tight junction: implication on inflammatory bowel diseases. Intest Res 13:11-18

\section{Submit your next manuscript to BioMed Central and we will help you at every step:}

- We accept pre-submission inquiries

- Our selector tool helps you to find the most relevant journal

- We provide round the clock customer support

- Convenient online submission

- Thorough peer review

- Inclusion in PubMed and all major indexing services

- Maximum visibility for your research

Submit your manuscript at www.biomedcentral.com/submit 